\title{
HERRAMIENTA PICO PARA LA FORMULACIÓN Y BÚSQUEDA DE PREGUNTAS CLÍNICAMENTE RELEVANTES EN LA PSICOONCOLOGÍA BASADA EN LA EVIDENCIA
}

\author{
PICO TOOL FOR THE FORMULATION AND SEARCH OF CLINICALLY RELEVANT \\ QUESTIONS IN EVIDENCE-BASED PSYCHO-ONCOLOGY
}

Edgar Landa-Ramírez y Aranel de Jesús Arredondo-Pantaleón

Facultad de Psicología/Programa de Medicina Conductual. Universidad Nacional Autónoma de México.

Resumen

El objetivo de este estudio fue proporcionar una descripción del sistema PICO, ejemplificar el uso del mismo para la formulación de preguntas clínicas relevantes y la búsqueda de artículos en el área de la psicooncología. A lo largo del escrito, se describen los principales elemento del sistema PICO y la forma en la cual se pueden integrar para formular preguntas clínicas relevantes. De igual forma, se ejemplifica por medio de un caso de la práctica psicooncológica diaria, la forma en la cual se desarrollan los pasos en PICO y las bases de datos en las cuales se pueden realizar la búsqueda de información, para solventar las preguntas planteadas. Finalmente, se describen las críticas a la herramienta y las posibles áreas de investigación en un futuro.

Palabras clave: Sistema PICO, práctica basada en la evidencia, preguntas clínicamente relevantes.

\section{Abstract}

The aim of this study was to provide a description of the PICO system, exemplify the use thereof for the formulation of clinically relevant questions and show how to search for papers in the field of psycho-oncology. Through this paper, the main component of PICO system and how that can be integrated to make relevant clinical questions are described. Using and example We show the way in which the psycho-oncologists can use de PICO system in their daily consultation. Finally, criticism of the tool and future research in the field are described.

Key words: PICO system, evidence-based practice, clinically relevant questions.
Datos recientes muestran que existe una transición epidemiológica del cáncer, la cual se caracteriza por la disminución de la enfermedad en países desarrollados y el incremento de la misma en países en vías de desarrollo(1). De seguir la tendencia actual, se calcula que para el año 2030, se tendrán 22 millones de nuevos casos de cánceres, de los cuales el 60\% ocurrirán en países en vías de desarrollo ${ }^{(2,3)}$.

\section{Correspondencia:}

Edgar Landa-Ramírez o Aranel de Jesús Arredondo

Universidad Nacional Autónoma de México.

Av. Universidad 3114 , Col. Copilco Universidad, Del. Coyoacán,

04510 México D.F., México, tel. (55)56-22-23-32,

E-mail: edgar_landa_ramirez@yahoo.com.mx y aranel_arredondo_pantaleon@yahoo.com.mx 
En América Latina, se calcula que para el año 2020, habrá un incremento de la incidencia anual del 33\%, lo que significa aproximadamente 17 millones de $\operatorname{casos}^{(4)}$.

Con base en este panorama epidemiológico, se puede prever que el psicooncólogo que vive en países en vías de desarrollo y particularmente en América Latina, se enfrentará a una mayor cantidad de pacientes, sin embargo, estará inmerso en una infraestructura insuficiente para atender la demanda de los mismos ${ }^{(4)}$. Es así, que el psicooncólogo necesitará desarrollar habilidades y competencias, que le permitan atender de manera expedita y eficiente, los distintos síntomas y problemas comportamentales, cognitivos, emocionales y familiares, tanto en los pacientes, como en los cuidadores primarios formales e informales; sorteando al mismo tiempo, las vicisitudes propias del sistema de salud al que pertenece.

Dada la cantidad actual de investigaciones, avances tecnológicos, tratamientos psicológicos y de publicaciones en donde se reportan, el psicooncólogo puede confundirse sobre qué criterios debe de seguir cuando elige la mejor intervención para tratar a un paciente o cuidador ${ }^{(5,6)}$ : el paradigma de la práctica basada en la evidencia podría ayudar al personal de psicología en el área de cáncer, a identificar tres criterios para aplicar intervenciones consensuadas por la comunidad científica en turno ${ }^{(6,7)}: 1$ ) la jerarquía de la investigación consultada. 2) condiciones institucionales junto con la experiencia del personal de salud y 3) los valores, preferencias, expectativas y metas de los pacientes.

Se define a la práctica basada en la evidencia como "el uso consciente, explícito y juicioso de las mejores y actuales pruebas en la toma de decisiones sobre el cuidado individual del paciente" ${ }^{(8,9)}$ y se considera que se debe de combinar el mejor nivel de evidencia de la investigación consultada, con los deseos del paciente, la experiencia del personal de salud y el contexto institucional en el que se ve al paciente, para poder tomar la mejor decisión clínica ${ }^{(10)}$.

De acuerdo con el Centro de Medicina Basada en la Evidencia de Oxford ${ }^{(11)}$, existen distintos niveles de evidencia, dependiendo del tipo de pregunta que se trate de contestar (prevalencia, diagnóstico, pronóstico, tratamiento, posibles daños y evaluaciones). En el caso de las preguntas que abordan los efectos de los tratamientos o intervenciones, se recomienda buscar y leer críticamente los siguientes tipos de estudios: revisiones sistemáticas, ensayos controlados aleatorizados, estudios de Cohorte, series de casos, casos y controles y mecanismos basados en el razonamiento. A partir de dichos estudios, se realizan jerarquías del conocimiento para guiar parte de la elección clínica que lleva a cabo el personal de salud, es así, que las revisiones sistemáticas y los ensayos controlados aleatorizados (nivel de evidencia 1 y 2) serán siempre las primeras opciones de elección. Los estudios de cohorte, series de casos, correlacionales y en general estudios analítico observacionales (nivel de evidencia 3 y 4) serán tomados con precaución y únicamente si no existen estudios con mayor nivel de evidencia. Finalmente, las opiniones de expertos, o los mecanismos basados en el razonamiento, serán la última elección que el psicooncólogo deberá de realizar (Nivel de evidencia 5; para una lectura amplia de las jerarquías del conocimiento, se recomienda consultar los escritos de Oxford, Glasziou et al. y Sackett et al. $)^{(9,11,12)}$.

Se recomienda que todo psicooncólogo realice cinco pasos básicos, que le permitan hacer una búsqueda y aplicación pertinente del conocimiento interno y externo que existe sobre el padecimiento de su interés para poder seleccionar el mejor tratamiento para atender a su paciente; los pasos recomendados a seguir son los si- 
guientes: 1) planteamiento de preguntas; 2) Localización de la mejor evidencia; 3) evaluación crítica de la evidencia obtenida; 4) integrar y aplicar la evidencia y 5) evaluar los resultados ${ }^{(12,13)}$.

Se ha reportado reticencia del personal de salud para aplicar los cinco pasos de la práctica basada en la evidencia, en algunos casos, porque existen dudas sobre la viabilidad de aplicar éstos ${ }^{(13)}$, en otros casos, por no seguir una estrategia concreta de cómo comenzar a plantear preguntas claras, fallando así, en el paso número uno y repercutiendo con el resto de los pasos ${ }^{(12)}$.

Lo anterior es un importante problema, porque si no existe una pregunta correctamente formulada, será muy difícil encontrar la información pertinente para atender el malestar de los pacientes ${ }^{(14)}$, por lo cual, es muy necesario desarrollar habilidades para poder llevar de manera correcta la formulación de preguntas y la localización de la mejor evidencia acorde a la pregunta planteada. Es por esta razón que se considera indispensable hacer una descripción de la herramienta PICO, la cual será útil para poder llevar a cabo los primeros dos pasos propuestos en la práctica basada en la evidencia, fomentando así, un mayor entendimiento del personal psicooncológico con los pasos iniciales del paradigma. Es así, que el objetivo de este estudio fue proporcionar una descripción del sistema PICO, ejemplificar el uso del mismo para la formulación de preguntas clínicas relevantes y la búsqueda de artículos en el área de la psicooncología.

\section{SISTEMA PICO}

La herramienta PICO constituye un formato especializado para desarrollar la pregunta clínica, es llamado así debido a que representa un acrónimo en inglés para describir los componentes que la integran, mismos que se describen en la tabla $1^{(12,16,17)}$.

Esta estrategia puede ser utilizada para construir diversos tipos de preguntas de investigación originadas de la práctica clínica, el manejo de recursos humanos y materiales o el uso de instrumentos de evaluación ${ }^{(16)}$. Frecuentemente se formulan preguntas sobre tratamiento o intervenciones, sin embargo, existen diversos tipos de cuestiones clínicas que pueden estructurarse mediante el sistema PICO, tales como: etiología y factores de riesgo, manifestaciones clínicas de la enfermedad,

\section{Tabla 1. Descripción de los componentes del sistema PICO.}

\begin{tabular}{|l|l|}
\hline \multicolumn{1}{|c|}{$\begin{array}{c}\text { Acrónimo y } \\
\text { componente }\end{array}$} & \multicolumn{1}{c|}{ Descripción de los componentes } \\
\hline $\begin{array}{l}\text { P. Paciente o } \\
\text { problema de interés } \\
\text { (Population) }\end{array}$ & $\begin{array}{l}\text { Paciente o grupo con una condición en particular, se trata de destacar } \\
\text { las características del paciente que puedan contribuir a delimitar la } \\
\text { búsqueda de información. }\end{array}$ \\
\hline $\begin{array}{l}\text { I. Intervención } \\
\text { (Intervention) }\end{array}$ & $\begin{array}{l}\text { Manejo o intervención de interés, misma que puede ser terapéutica, } \\
\text { preventiva, diagnóstica o de pronóstico. }\end{array}$ \\
\hline $\begin{array}{l}\text { C. Comparación } \\
\text { (Comparison) }\end{array}$ & $\begin{array}{l}\text { Se trata de una intervención alternativa con la cual comparar, aunque no } \\
\text { siempre se dispone de la misma, en cuyo caso se omite este componente } \\
\text { y el formato se convierte en PIO. }\end{array}$ \\
\hline $\begin{array}{l}\text { O. Resultados } \\
\text { (Outcome) }\end{array}$ & $\begin{array}{l}\text { Se trata de las consecuencias relevantes de interés, el resultado esperado } \\
\text { de la intervención. }\end{array}$ \\
\hline
\end{tabular}


diagnóstico diferencial, frecuencia de un problema específico, métodos y herramientas de diagnóstico y pronóstico ${ }^{(12,18)}$.

La formulación de preguntas con la estrategia PICO sirve para mejorar la especificidad y claridad conceptual de los problemas clínicos a estudiar, así como para realizar búsquedas que arrojen resultados con mayor calidad y precisión. Asimismo, permite maximizar la localización de información relevante, focalizando los objetivos de la búsqueda y rechazando la información innecesaria, lo cual ayudará a tomar las mejores decisiones basadas en la evidencia ${ }^{(15,19,20)}$.

A continuación se presenta un ejemplo aplicado al campo de la psicooncología para ilustrar el desarrollo de la pregunta basada en este formato: paciente femenino de 51 años con diagnóstico oncológico de cáncer de mama en estadio IV, con metástasis a hueso y pulmón, dado el estado de la paciente, va a recibir tratamiento médico enfocado únicamente al control de síntomas y se calcula que tiene una expectativa de vida menor a seis meses. Es referida al área de psicooncología por el médico tratante, debido a que presenta ansiedad, miedo, además de que no puede estar calmada y tranquila. Dada las recomendaciones clínicas actuales basadas en la evidencia ${ }^{(21)}$, el personal de psicología decide aplicar terapia cognitivo conductual para abordar la ansiedad de la paciente, pero se preguntan si es conveniente -para optimizar tiempoel aplicar este tipo de terapia de manera grupal (con otros pacientes con cáncer terminal) o es necesario hacerlo de manera individual. Siguiendo la estrategia PICO, los diferentes componentes quedarían desglosados como se muestra a continuación:

\section{P. Paciente con cáncer de mama en esta- dio IV}

I. Terapia cognitivo conductual grupal

C. Terapia cognitivo conductual individual

O. Disminución de la ansiedad
La estructura que sugiere PICO para formular la pregunta es la siguiente: ¿para "P" es igual de efectivo "I" que "C" para conseguir " $\mathrm{O}$ "? En términos del ejemplo planteado, la pregunta sería la siguiente oración: ¿para una paciente con cáncer de mama en estadio IV, es igual de efectiva la terapia cognitivo conductual grupal que la terapia cognitivo conductual individual para disminuir la ansiedad?

Siguiendo el mismo ejemplo, supóngase que un miembro del equipo de psicooncología, refiere haber leído una revisión sistemática con meta-análisis reciente, en la que se reporta que la relajación es una técnica con muy buenos efectos para disminuir la ansiedad en los pacientes ${ }^{(22)}$, por lo cual se pregunta, si será suficiente tratar a la paciente con relajación para disminuir la ansiedad que presenta.

Como podrá observarse, en este ejemplo no se presenta un tipo de intervención con el cual comparar la relajación, por lo que en este caso, se omite el componente " $\mathrm{C}$ ", quedando el formato como PIO. Por lo anterior, la estructura para formular la pregunta será ¿Para "P" es efectivo "I" para conseguir "O"?, por lo que en términos del ejemplo, la pregunta formulada será: ¿para una paciente con cáncer de mama en estadio IV, es efectiva la relajación para disminuir la ansiedad?

Los componentes quedarían desglosados de la siguiente forma:

P. Paciente con cáncer de mama en estadio IV

I. Técnicas de relajación

O. Disminución de la ansiedad

Ahora bien ¿cómo responder a esas preguntas a través de la búsqueda de evidencia en la literatura científica?

Se sugiere realizar una descripción de diferentes sinónimos o palabras relacionadas con los términos de cada elemento de PICO y para ello pueden buscarse en inter- 
net los Encabezados de Temas Médicos (en inglés, MeSH Headings) que representan una lista de términos controlados que proporciona uniformidad al vocabulario utilizado para indexar artículos en las bases de datos, describiendo el contenido de los mismos a través de palabras clave. Asimismo, conducen, a partir de la lógica de la pregunta formulada, a las categorías ramificadas para cada componente de $\mathrm{PICO}^{(23)}$.

Retomando el caso clínico, en las tablas 2 y 3, se pueden observar las palabras clave relacionadas con cada elemento de $\mathrm{PICO}$ y $\mathrm{PIO}$.

Una vez formulada la pregunta y habiendo identificado sus diversos componentes y palabras clave, el profesional de la psicooncología debe realizar la búsqueda de la literatura más adecuada para responder su interrogante y para ello existen diversos recursos e interfaces en la red. A continuación se describen con brevedad las más utilizadas en el área de la psicooncología.

Tabla 2. Palabras clave relacionadas con cada elemento de PICO.

\begin{tabular}{|c|c|c|c|}
\hline \multicolumn{4}{|l|}{ Palabras clave } \\
\hline $\mathrm{P}$ & 1 & C & $\mathrm{O}$ \\
\hline $\begin{array}{l}\text { Advanced cancer } \\
\text { Cancer stage IV } \\
\text { Palliative cancer } \\
\text { Terminal cancer } \\
\text { Metastatic cancer }\end{array}$ & $\begin{array}{l}\text { Group Cognitive behavioral } \\
\text { therapy } \\
\text { Psychotherapy } \\
\text { CBT } \\
\text { Behavioral therapy } \\
\text { Cognitive therapy } \\
\text { Cognitive Psychotherapy }\end{array}$ & $\begin{array}{l}\text { Individual Cognitive } \\
\text { behavioral therapy } \\
\text { Psychotherapy } \\
\text { CBT } \\
\text { Behavioral therapy } \\
\text { Cognitive therapy } \\
\text { Cognitive Psychotherapy }\end{array}$ & $\begin{array}{l}\text { Anxiety } \\
\text { Distress } \\
\text { Catastrophization } \\
\text { Fear }\end{array}$ \\
\hline
\end{tabular}

Tabla 3. Palabras clave relacionadas con cada elemento de PIO.

\begin{tabular}{|l|l|l|}
\hline \multicolumn{2}{|l|}{ Palabras clave } & O \\
\hline $\mathbf{P}$ & Relaxation therapy & Anxiety \\
\hline Advanced cancer & Progressive muscle relaxation & Distress \\
training & Catastrophization \\
Cancer stage IV & Guided imagery training & Fear \\
Palliative cancer & & \\
Terminal cancer & Autogenic relaxation & \\
Metastatic cancer & Pursed-lips breathing & \\
& Applied relaxation and meditation & \\
&
\end{tabular}




\section{BÚSQUEDA DE LA INFORMACIÓN}

La base de datos más utilizada es Medline, que depende de la Biblioteca Nacional de Medicina de los Estados Unidos (NLM). Es una de las bases más completas de su área, procesando anualmente un aproximado de 4.800 títulos de publicaciones seriadas que proceden de más de 70 países y alcanza 15 millones de referencias de trabajos publicados desde la década de los años 60 hasta la fecha ${ }^{(14,24)}$. Se accede a ella por medio de PubMed, un proyecto impulsado por el Centro Nacional para la Información Biotecnológica (NCBI), cuyo objetivo es facilitar el acceso a los textos completos tanto de Medline, como de otras bases de datos afines recopiladas por la $\mathrm{NLM}^{(25)}$. Para iniciar la búsqueda en la base de datos Medline a través de PubMed es necesario acceder a la página http://www. ncbi.nlm.nih.gov/pubmed y para tener acceso a un tutorial extenso acerca de cómo efectuar búsquedas, se sugiere acceder a la siguiente dirección electrónica: http:// www.nlm.nih.gov/bsd/disted/pubmed.html

CINAHL es una base de datos diseñada especialmente para los profesionales de la enfermería, la fisioterapia y la terapia ocupacional, disciplinas de las cuales proporciona todas las revistas disponibles en inglés. Cuenta con un equivalente impreso que es el Índice Acumulativo de Enfermería y Literatura Relacionada con la Salud (Cumulative Index to Nursing \& Allied Health Literature). La importancia y utilidad de esta base de datos radica en el área de la enfermería se llevan a cabo estudios valiosos y de calidad en temas relacionados con las ciencias del comportamiento, por lo que resulta de gran provecho consultar dicha base de datos para conocer los artículos que ofrezcan mayor nivel de evidencia relacionado con la pregunta de investigación que se esté buscando contes$\operatorname{tar}^{(26)}$. Para acceder a la página de CINAHL se escribe la dirección electrónica http:// www.ebscohost.com/nursing/products/ cinahl-databases/cinahl-complete y se puede consultar un tutorial sobre su uso en: http://es.scribd.com/doc/55087570/ Tutorial-Cinahl-base-de-datos-EBSCO

Psyclnfo es una base de datos especializada en psicología, desarrollada por la Asociación Americana de Psicología (APA), cuenta con más de 3,6 millones de registros de revistas especializadas, libros y disertaciones que además de psicología, abarca disciplinas afines como la medicina, el trabajo social, las neurociencias o la enfermería ${ }^{(27)}$. Se accede mediante la siguiente dirección: http://www.apa.org/ pubs/databases/psycinfo/index.aspx

La Red de Revistas Científicas de América Latina y el Caribe, España y Portugal (Redalyc) es una iniciativa académica de la Universidad Autónoma del Estado de México, de acceso gratuito que tiene como finalidad la divulgación de documentos científicos provenientes de las regiones ya mencionadas $^{(28)}$. Resulta prudente mencionar esta base de datos, ya que debido a las regiones que abarca, se puede obtener evidencia enfocada a las características sociodemográficas específicas de la población iberoamericana. Para buscar en esta base de datos se escribe la siguiente dirección http://www.redalyc.org/home.oa

La colaboración Cochrane es una base de datos que brinda un índice de revisiones sistemáticas desarrolladas a partir de ensayos clínicos aleatorizados, ofreciendo información ya revisada a partir de artículos con nivel de evidencia de 1, por lo cual resulta ampliamente recomendable su uso ${ }^{(20)}$. Para ingresar a Cochrane, se utiliza la siguiente dirección http://www. cochrane.org/cochrane-reviews

Al acceder a ellas, se puede realizar una búsqueda simple introduciendo en la casilla los términos formulados en la pregunta de investigación (descriptores), enlazados mediante operadores booleanos, mismos que permiten enfocar la búsqueda 
vinculando los términos y definiendo la relación entre ellos ${ }^{(25)}$. El operador booleano de combinación, AND, permitirá localizar registros que contengan todos los términos solicitados (continuando con el ejemplo, si se introduce terminal cancer AND anxiety, la búsqueda arrojará todos los resultados que contengan ambos términos). El operador de suma, OR es útil para localizar evidencia que contenga cualquiera o todos los descriptores (si se introduce terminal cancer OR anxiety, la búsqueda arrojará resultados que tengan los términos terminal cancer, anxiety o ambos). El operador NOT es de exclusión y permite localizar la información que contenga el primer tér- mino, pero no el segundo (si escribimos terminal cancer NOT anxiety, la búsqueda arrojará resultados sobre cáncer, pero no incluirá evidencia sobre ansiedad relacionada con cáncer) ${ }^{(15,16)}$. La recuperación de los estudios más relevantes puede mejorar notablemente mediante estas combinaciones de términos y palabras de texto ${ }^{(29)}$. Los operadores booleanos se escriben siempre en mayúscula, si se desea una búsqueda muy específica, se pueden entrecomillar los término clave ${ }^{(25,29,30)}$.

Siguiendo con el ejemplo, en la tabla 4 se presentan algunos posibles cruces de palabras clave enlazadas con el operador booleano AND para efectuar la búsque-

\section{Tabla 4. Ejemplo de posibles cruces de términos componentes de PICO para efectuar la búsqueda en bases de datos.}

\begin{tabular}{|l|c|c|c|c|c|}
\hline \multicolumn{5}{|c|}{ Secuencia de la búsqueda } \\
\hline Cruces de tres palabras & $\begin{array}{c}\text { Artículos } \\
\text { localizados } \\
\text { Pubmed }\end{array}$ & $\begin{array}{c}\text { Artículos } \\
\text { localizados } \\
\text { CINAHL }\end{array}$ & $\begin{array}{c}\text { Artículos } \\
\text { localizados } \\
\text { Psyclnfo }\end{array}$ & $\begin{array}{c}\text { Artículos } \\
\text { localizados } \\
\text { Redalyc }\end{array}$ & $\begin{array}{c}\text { Artículos } \\
\text { localizados } \\
\text { Cochrane }\end{array}$ \\
\hline $\begin{array}{l}\text { Advanced cancer AND Cognitive } \\
\text { behavioral therapy AND Anxiety }\end{array}$ & 33 & 0 & 1 & 299 & 1 \\
\hline $\begin{array}{l}\text { Advanced cancer AND Cognitive } \\
\text { therapy AND Anxiety }\end{array}$ & 33 & 5 & 2 & 299 & 0 \\
\hline $\begin{array}{l}\text { Advanced cancer AND } \\
\text { Psychotherapy AND Anxiety }\end{array}$ & 51 & 9 & 2 & 182 & 1 \\
\hline $\begin{array}{l}\text { Advanced cancer AND Behavioral } \\
\text { therapy AND Anxiety }\end{array}$ & 50 & 0 & 1 & 293 & 0 \\
\hline \multicolumn{2}{|c|}{ Ejemplo de posibles cruces de términos componentes de PIO para efectuar la búsqueda en } \\
\hline $\begin{array}{l}\text { Advanced cancer AND Relaxation } \\
\text { therapy AND Anxiety }\end{array}$ & 15 & 0 & 0 & 69 & 0 \\
\hline $\begin{array}{l}\text { Advanced cancer AND Relaxation } \\
\text { AND Anxiety }\end{array}$ & 16 & 5 & 2 & 83 & 0 \\
\hline $\begin{array}{l}\text { Advanced cancer AND Progressive } \\
\text { muscle relaxation training AND } \\
\text { Anxiety }\end{array}$ & 2 & 1 & 0 & 40 & 0 \\
\hline $\begin{array}{l}\text { Advanced cancer AND Guided } \\
\text { imagery training AND Anxiety }\end{array}$ & 2 & 1 & 0 & 2 \\
\hline
\end{tabular}


da, en el que se registró el número de resultados a partir de la búsqueda en los últimos años, arrojados por cada una de las bases de datos descritas en el recuadro correspondiente. En el ejemplo se utilizó únicamente el operador booleano AND, no obstante, lo recomendable es efectuar la búsqueda con todos los operadores booleanos descritos en el presente artículo y efectuando todos los posibles cruces de palabras para recuperar la mayor cantidad posible de resultados.

Es muy importante mencionar, que en los buscadores de Psyclnfo y Redalyc, es indispensable delimitar de manera clara los criterios de búsqueda (buscar en el título, resumen o palabras claves) de lo contrario estos buscadores despliegan una cantidad enorme de artículos los cuales dificultan el trabajo de identificación de aquellos que son pertinentes. Una vez realizada la búsqueda, se deben de seleccionar aquellos artículos que sí aborden el tema de interés y que proporcionen un mayor nivel de evidencia, (revisiones sistemáticas y ensayos clínicos aleatorizados) en caso de no contar con este tipo de resultados, se elegirán estudios con menor nivel de evidencia, aunque debe tenerse cuidado ya que a medida que el nivel de evidencia baja, también se vuelven menos recomendables los estudios. Una vez seleccionados los artículos, se procede a efectuar lectura crítica del artículo que permita determinar la validez interna de la investigación y así decidir si pueden ser utilizados para contestar a la pregunta formulada. Con base en la información seleccionada, el personal de salud podrá juntar los elementos necesarios para tomar la decisión clínica de si es pertinente o no tratar al paciente con la información obtenida.

Siguiendo con nuestro ejemplo, después de realizar la búsqueda y llevar a cabo la lectura crítica de los artículos, los psicooncólogos retomarían 4 estudios controlados aleatorizados que evaluaron el efecto de la terapia cognitivo conductual grupal e individual sobre la ansiedad en pacientes con cáncer avanzado ${ }^{(31-34)}$, ambas modalidades de terapias mostraron tener efectos positivos para disminuir la ansiedad, sin embargo, la TCC individual, mostró tener mejor tamaño del efecto de la intervención para disminuirla (valor d de Cohen $=0,80)^{(34)}$, además de que un estudio de TCC grupal, reportó que tuvo complicaciones emocionales no previstas en los pacientes que asistían a la terapia, ya que antes de que terminara la misma, fallecieron un par de pacientes, lo cual afectó el estado de ánimo del grupo ${ }^{(31)}$.

En el caso de la relajación, se encontró un estudio controlado aleatorizado que evaluó el efecto de diversas técnicas de relajación sobre la ansiedad en pacientes con cáncer avanzado ${ }^{(35)}$, sin embargo, al realizarse la lectura crítica del artículo, se encontró que el artículo no reportó información básica del estudio, (número de participantes por grupo, tipo de aleatorización empleada, muerte experimental entre evaluaciones) lo cual influyó en que no se pudiera tomar una decisión con respecto a la eficacia de la técnica sola para el control de la ansiedad en pacientes con cáncer terminal.

A partir de la evidencia obtenida, el psicooncólogo podría identificar que la TCC individual es de las mejores opciones psicoterapéuticas para tratar a la paciente descrita. Es así, que el psicooncólogo se encargaría de explicar, adaptar y aplicar los 4 componentes utilizados en la terapia para esta población (psico-educación, relajación, reestructuración de pensamientos y planeación de actividades) a las características particulares de la paciente.

Finalmente, siguiendo los pasos de la práctica basada en la evidencia, el personal de psicooncología podría evaluar si el tratamiento propuesto, se adecua a los intereses de la paciente y se valoraría la factibilidad de aplicar esta terapia en el 
contexto en el cual ambos están inmersos; en caso de que sea factible aplicar el tratamiento, el psicooncólogo tendrá que valorar posteriormente los efectos específicos que la terapia tuvo en la paciente que atendió.

El proceso descrito constituye un ejemplo de cómo tomar una decisión clínica congruente con los pasos de la práctica basada en la evidencia para tratar un problema en el ámbito oncológico, además de que ilustra el uso de la herramienta PICO para la formulación clara de los elementos que conformaban el problema clínico en turno.

\section{CONSIDERACIONES FINALES}

El objetivo de este estudio fue proporcionar una descripción del sistema PICO, ejemplificar el uso del mismo para la formulación de preguntas clínicas relevantes y la búsqueda de artículos en el área de la psicooncología. Como se ha descrito, el sistema PICO, es considerado una herramienta ampliamente recomendable para comenzar correctamente los pasos de la práctica basada en la evidencia ${ }^{(14)}$, sin embargo, es necesario entrenar a las personas en su uso, ya que un par de estudios han demostrado, que el personal de salud no entrenado en esta herramienta, realiza una gran cantidad preguntas clínicas con una mínima estructura, lo cual mengua la precisión de su búsqueda(19,36).

Aunado a lo anterior, investigaciones recientes han comentado que una significativa cantidad de resúmenes de la literatura en el área de la salud, se encuentran desestructurados o poco estructurados, lo que dificulta la tarea de encontrar rápidamente los componentes de $\mathrm{PICO}^{(37-39)}$, razón por la cual, se ha comenzado a evaluar el uso de diferentes algoritmos informáticos para hacer más precisa la búsqueda de los componentes en bases de datos científicas. Al utilizar los algoritmos, se han encontrado diferencias para identificar el elemento " $\mathrm{P}$ "(37,38) no así cuando se tratan de identificar los elementos "I" $\mathrm{y}$ "O"(38). Comentan los autores que esto se podría deber a que en las bases de datos electrónicas, el elemento "I" puede caer en varias clasificaciones, por ejemplo: un medicamento puede clasificarse en marcas comerciales o sustancias activas; en el caso de la psicooncología, una terapia puede clasificarse en la categoría psicoterapias o en terapias específicas, (terapia sistémica, terapia existencial, terapia de aceptación y compromiso) lo cual dificulta aún más su búsqueda, el psicooncólogo deberá de ser muy exhaustivo, a la hora de considerar las palabras clave que buscará en los componentes "I" " $\mathrm{C}$ " $\mathrm{y}$ " $\mathrm{O}$ ".

Es muy importante mencionar, que existen muy pocos estudios que proporcionen datos empíricos de que es mejor usar el sistema PICO que otro tipo de sistema similar; el realizar este tipo de estudios es indispensable, ya que resulta paradójico que la herramienta que se promociona como la clave para comenzar correctamente con los pasos que guían a la practica basada en la evidencia, no tenga tantos estudios que den evidencia de su uso. El problema, es común en el paradigma de la práctica basada en la evidencia, ya que varios de los pasos que propone realizar, no presentan datos de su superioridad al usarlos $^{(40,41)}$.

Recientemente, algunos autores han señalado que el buscador PICO, es una opción viable para atender preguntas sobre intervenciones, pero mucho menos viable para preguntas de diagnóstico, pronóstico o etiología(19), razón por la cual, se han desarrollado herramientas derivadas de PICO, más complejas, en donde se agregan un par de elementos, que permiten realizar una búsqueda más específica para este tipo de preguntas ${ }^{(42)}$. De igual forma, se ha señalado el efecto limitado de PICO para identificar estudios de índole cuali- 
tativo, por lo que se han propuesto otro tipos de herramientas para realizar dichas búsquedas ${ }^{(43)}$.

Independientemente de las críticas antes mencionadas, los autores de este escrito, consideramos que la herramienta $\mathrm{PICO}$, es una opción útil para que el personal de psicooncología tome las decisiones clínicas que actualmente se le presentan y para que se prepare para aquellos retos que se anticipa tendrá que afrontar en un futuro no muy lejano, razón por la cual recomendamos ampliamente el uso de esta herramienta.

\section{REFERENCIAS BIBLIOGRÁFICAS}

1. Jemal A, Center MM, DeSantis C, Ward EM. Global patterns of cancer incidence and mortality rates and trends. Cancer Epidemiol Biomarkers Prev 2010;19:1893-907. Doi: 10.1158/1055-9965.EPI-10-0437.

2. Bray F, Jemal A, Grey N, Ferlay J, Forman D. Global cancer transitions according to the Human Development Index (20082030): A population-based study. Lancet Oncol 2012;13:790-801. Doi:10.1016/ S1470-2045(12)70211-5.

3. Jemal A, Bray F, Center MM, Ferlay J, Ward E, Forman D. Global cancer statistics. CA Cancer J Clin 2011;61:6990. Doi:10.3322/caac.20107.

4. Goss PE, Lee BL, Badovinac-Crnjevic T, Strasser-Weippl K, Chavarri-Guerra Y, St Louis J, et al. Planning cancer control in Latin America and the Caribbean. Lancet Oncol 2013;14:391-436. Doi: 10.1016/ S1470-2045(13)70048-2.

5. Zietman A, Ibbott G. A clinical approach to technology assessment: How do we and how should we choose the right treatment? Semin Radiat Oncol 2012;22:11-7. Doi: 10.1016/j.semradonc.2011.09.008.

6. Jacobsen PB. Promoting evidence-based psychosocial care for cancer patients. Psychooncology 2009;18:6-13. Doi: 10.1002/pon.1468.
7. Montori VM, Guyatt GH. Progress in evidence-based medicine. JAMA 2008; 300:1814-6. Doi: 10.1001/ jama.300.15.1814.

8. Evidence-Based Medicine Working Group. Evidence-based medicine: A new approach to teaching the practice of medicine. JAMA 1992;268:2420-5. Doi:10.1001/ jama.1992.03490170092032

9. Sackett DL, Rosenberg WM, Gray JA, Haynes RB, Richardson WS. Evidence based medicine: What it is and what it isn't. BMJ 1996;312:71-2. Doi: 10.1136/ bmj.312.7023.71

10. Haynes RB, Devereaux PJ, Guyatt GH. Physicians' and patients' choices in evidence based practice. BMJ 2002;324:1350. Doi: 10.1136/bmj.324.7350.1350

11. OCEBM levels of evidence working group. The Oxford 2011 levels of evidence. Oxford Centre for Evidence-Based Medicine. En línea. [Acesso 10 de mayo de 2014]. Disponible en: http://www. cebm.net/index.aspx?o=5653

12. Glasziou P, Del Mar C, Salisbury J. Evidence-based medicine workbook: Finding and applying the best research evidence to improve patient care. London: BMJ Books; 2003.

13. Parzsolt F, Ochletz A, Thim A, Gardner D. Evidence-based decision makingthe six step approach. Evid Based Med 2003;8:165-6. Doi.org/10.1136/ ebm.8.6.165.

14. Schardt C, Adams MB, Owens T, Keitz $\mathrm{S}$, Fontelo P. Utilization of the PICO framework to improve searching PubMed for clinical questions. BMC Med Inform Decis Mak 2007;7:16. Doi:10.1186/14726947-7-16.

15. Akobeng A. Principles of evidence based medicine. Arch Disease Child 2005; 90:837-40. Doi:10.1136/adc.2005.071761

16. Mamédio C, Andrucioli M, Cuce M. The PICO strategy for the research question construction and evidence research. Rev Latino-Am Enfermagem 2007;15:508-11. 
17. Ackley R. Evidence - based medicine. Univ Conn Health Cent 1999;8:1-8.

18. Virgilio R, Chiapa A, Palmarozzi E. Evidence-Based Medicine, Part 1. An introduction to creating an answerable question and searching the evidence. JAOA 2007; 8:295-7.

19. Huang X, Lin J, Demmer D. Evaluation of PICO as a knowledge representation for clinical questions. AMIA Annu Symp Proc 2006; 2006:369-3.

20. Ibáñez P, Alapont M. Introducción a la medicina basada en la evidencia. Cir Pediatr 2005;18:55-60.

21. Traeger L, Greer JA, Fernandez-Robles C, Temel JS, Pirl WF. Evidence-based treatment of anxiety in patients with cancer. J Clin Oncol 2012;30:1197-205. Doi: 10.1200/JCO.2011.39.5632.

22. Manzoni GM, Pagnini F, Castelnuovo G, Molinari E. Relaxation training for anxiety: A ten-years systematic review with metaanalysis. BMC Psychiatry 2008;8:41. Doi: $\quad 10.1186 / 1471-244 X-8-41$. Doi: 10.1186/1471-244X-8-41

23. PubMed en español. National Library of Medicine Madrid: Biblioteca de medicina de la Universidad Autónoma de Madrid [actualizado octubre 2010]. En línea. [Acceso el 24 de febrero de 2014]. Disponible en http://biblioteca.uam.es/ medicina/PubMedFormacion/index.htm

24. Castillo A, Rodrigo L, Sahores S, Urresti A. Utilización de bases de datos y revistas médicas online por médicos no docentes de la Ciudad de Corrientes. Rev Posg Vía Cát Med 2007;172:1-3

25. Betheshda. PubMed Help, NCBI Help Manual [Internet]. Estados Unidos de América: National Center for Biotechnology Information, U.S National Library of Medicine; 2005. En línea. [Acceso 2 sept 2013]. Disponible en: https://www.ncbi.nlm.nih.gov/books/ NBK3830/?report=printable

26. Ebscohost.com [Internet]. EBSCO Host [Actualizado 2014; acceso el 22 de febrero de 2014]. Disponible en: http:// www.ebscohost.com/nursing/products/ cinahl-databases/cinahl-complete

27. Apa.org [Internet]. Washington, DC: American Psychological Assotiation. En línea. [2014; acceso el 22 de febrero de 2014]. Disponible en: http://www.apa.org/ pubs/

28. Redalyc. org [Internet]. Estado de México: Red de Revistas Científicas de América Latina y el Caribe, España y Portugal. Sistema de Información Científica. Universidad Autónoma del Estado de México. En línea. [2013; acceso el 22 de febrero de 2014]. Disponible en http://www.redalyc.org/ home.oa

29. Haynes R, et al. Developing optimal search. Strategies for detecting clinically sound studies in MEDLINE. J Med Libr Assoc 1994; 1:447-58.

30. Heneghan C, Badenoch D. Evidencebased Medicine Toolkit. 2nd ed. Oxford: BMJ Books; 2006.

31. Edelman S, Bell DR, Kidman AD. A group cognitive behaviour therapy programme with metastatic breast cancer patients. Psychooncology 1999;8:295-305. Doi:10.1002/(SICI)10991611 (199907/08)8:4\%3C295::AIDPON386\%3E3.3.CO;2-P

32. Savard J, Simard S, Giguère I, Ivers $H$, Morin CM, Maunsell E, et al. Randomized clinical trial on cognitive therapy for depression in women with metastatic breast cancer: Psychological and immunological effects. Palliat Support Care 2006;4:21937. Doi:10.1017/S1478951506060305

33. Moorey S1, Cort E, Kapari M, Monroe B, Hansford P, Mannix K, et al. A cluster randomized controlled trial of cognitive behaviour therapy for common mental disorders in patients with advanced cancer. Psychol Med 2009;39:713-23. Doi: $10.1017 /$ S0033291708004169.

34. Greer JA, Traeger L, Bemis H, Solis J, Hendriksen ES, Park ER, et al. A pilot randomized controlled trial of brief 
cognitive-behavioral therapy for anxiety in patients with terminal cancer. Oncologist 2012;17:1337-45. Doi: 10.1634/ theoncologist.2012-0041.

35. Sloman R. Relaxation and imagery for anxiety and depression control in community patients with advanced cancer. Cancer Nurs 2002;25:432-5. Doi:10.1097/00002820200212000-00005

36. Bergus GR, Randall CS, Sinift SD, Rosenthal DM. Does the structure of clinical questions affect the outcome of curbside consultations with specialty colleagues? Arch Fam Med 2000;9:541-7. Doi: 10.1001/archfami.9.6.541

37. Huang KC, Chiang IJ, Xiao F, Liao CC, Liu CC, Wong JM. PICO element detection in medical text without metadata: Are first sentences enough? J Biomed Inform 2013;46:940-6. Doi: 10.1016/j.jbi.2013.07.009

38. Demner-Fushman D, Lin J. Answering clinical questions with knowledge-based and statistical techniques. Computational Linguistics 2007;33:63-103. Doi:10.1162/ coli.2007.33.1.63
39. Boudin F, Nie JY, Bartlett JC, Grad R, Pluye $P$, Dawes M. Combining classifiers for robust PICO element detection. MC Med Inform Decis Mak 2010;15:10-29. Doi: 10.1186/1472-6947-10-29.

40. Goodman NW. Who will challenge evidence-based medicine? J R Coll Physicians Lond 1999;33:249-51.

41. ConcatoJ, Shah N, Horwitz RI. Randomized, controlled trials, observational studies, and the hierarchy of research designs. $\mathrm{N}$ Engl J Med 2000;342:1887-92. Doi:10.1056/ NEJM200006223422507

42. Dawes M, Pluye P, Shea L, Grad R, Greenberg A, Nie JY. The identification of clinically important elements within medical journal abstracts: Patient-Population-Problem, ExposureIntervention, Comparison, Outcome, Duration and Results (PECODR). Inform Prim Care 2007;15:9-16.

43. Cooke A, Smith D, Booth A. Beyond PICO: The SPIDER tool for qualitative evidence synthesis. Qual Health Res 2012;22:143543. Doi: 10.1177/1049732312452938. 